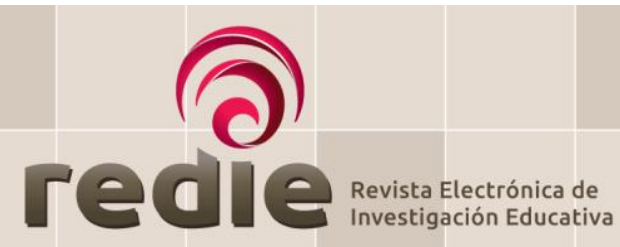

ISSN: $1607-4041$

https://redie.uabc.mx

Vol. 23, 2021/e05

\title{
Relación educativa entre profesor y educador tradicional en la educación intercultural bilingüe ${ }^{1}$
}

\section{Educational Relationship Between Teacher and Traditional Educator in Bilingual Intercultural Education}

Katerin Arias-Ortega (*) https://orcid.org/0000-0001-8099-0670

Segundo Quintriqueo $\left(^{*}\right.$ https://orcid.org/0000-0002-7228-4095

(*) Universidad Católica de Temuco

(Recibido: 1 de febrero de 2019; Aceptado para publicación: 1 de agosto de 2019)

Cómo citar: Arias-Ortega, K. y Quintriqueo, S. (2021). Relación educativa entre profesor y educador tradicional en la educación intercultural bilingüe. Revista Electrónica de Investigación Educativa, 23, e05, 1-14. https://doi.ora/10.24320/redie.2021.23.e05.2847

\section{Resumen}

El objetivo del artículo es exponer los desafíos de la relación educativa entre profesor mentor y educador tradicional en la implementación de la Educación Intercultural Bilingüe (EIB) en La Araucanía, Chile. Estos desafíos tienen relación con la incorporación de los aportes epistémicos de métodos, saberes y conocimientos educativos mapuches en la enseñanza de la lengua y la cultura mapuche en la EIB. La articulación de los aportes epistémicos mapuches y escolares permite una relación educativa intercultural caracterizada por la implicación mutua para avanzar hacia un diálogo de saberes inter-epistémicos en la escuela. La metodología utilizada es la investigación educativa desde un enfoque de complementariedad metodológica cualitativo-cuantitativo. Los resultados de la investigación muestran que existe una base de conocimientos educativos mapuches presente en la memoria social de los educadores tradicionales -que es pertinente incorporar al currículo escolar desde un enfoque educativo intercultural.

Palabras clave: relaciones interétnicas, conocimientos tradicionales, métodos educativos

\begin{abstract}
The objective of this article is to outline the challenges in the educational relationship between mentor teachers and traditional educators in the implementation of bilingual intercultural education (EIB, in Spanish) in Araucanía, Chile. These challenges concern the inclusion of epistemic contributions from Mapuche educational knowledge, methods and wisdom in teaching the Mapuche language and culture in EIB. Establishing links between academic and Mapuche epistemic contributions enables an intercultural educational relationship defined by mutual engagement to move toward a dialogue of inter-epistemic knowledge in school. The study employs educational research based on a mixed-method methodological complementarity approach. The results reveal a Mapuche educational knowledge base present in the social memory of traditional educators, which it is appropriate to integrate into the school curriculum from an intercultural educational approach.
\end{abstract}

\footnotetext{
${ }^{1}$ Investigación realizada gracias a los proyectos: PROFONDECYT 2019PF-KA-05, financiado por la Vicerrectoría de Investigación y Posgrado de la Universidad Católica de Temuco; FONDECYT DE INICIACIÓN N 11200306 y FONDECYT REGULAR N 1181531, financiados por la Agencia Nacional de Investigación y Desarrollo de Chile.
} 
Keywords: interethnic relationship, traditional knowledge, educational methods

\section{Introducción}

La educación escolar se ha abordado históricamente desde un enfoque de monoculturalidad eurocéntrica occidental, por lo que transmite sólo una cultura en el territorio nacional para la formación de las nuevas generaciones de indígenas y no indígenas (Quintriqueo et al., 2016). El sistema educativo chileno se organiza en cuatro niveles: Educación Parvularia, Educación Básica, Educación Media y Educación Superior. La Educación Parvularia atiende a la primeria infancia, desde los primeros meses de vida hasta el ingreso a la Educación Básica (Caiceo, 2011); la Educación Básica implica ocho años de formación basada en un currículum escolar único para todo el país (Ministerio de Educación de Chile [Mineduc], 2016); la Educación Media se desarrolla en cuatro años lectivos y brinda a los estudiantes la posibilidad de escoger entre una Educación Técnico-Profesional y una Educación Científico-Humanista (Mineduc, 2016); y por último, la Educación Superior se ofrece en Centros de Formación Técnica (CFT), Instituto Profesional (IP) y Universidades (Gaete y Morales, 2011). En este conglomerado de instituciones la universidad se encuentra en la cúspide de la pirámide educativa y ofrece dos tipos de programas de pregrado: técnico-profesional y licenciatura (Mineduc, 2016).

Desde principios del siglo XX en Latinoamérica se han implementado políticas de educación intercultural que han buscado avanzar en el reconocimiento y valoración de la diversidad cultural, lingüística y étnica al interior de los sistemas educativos (Dietz, 2014). Esta política de educación intercultural en Latinoamérica en general, se ha propuesto como finalidad educativa, contribuir en contrarrestar las desigualdades en educación, mediante la enseñanza de la lengua y la cultura propia, para luchar contra el racismo, la discriminación y exclusión, característicos en los contextos indígenas (Castillo y Caicedo, 2010). Esto ha posibilitado la habilitación de programas especiales para la formación de profesores de origen indígena y no indígena con el propósito de contar con profesionales capaces de brindar una educación intercultural, contextualizada y coherente con las necesidades de los pueblos indígenas (Gasché, 2013; Quintriqueo y Quilaqueo, 2019).

En ese contexto, según Fajardo (2011), en Latinoamérica se identifican tres etapas de desarrollo de la educación intercultural: 1) surge como una alternativa para contrarrestar la política de castellanización implementada por los Estados (López y Küper, 1999). En el caso mapuche, la apropiación del castellano tenía como objetivo defenderse de la usurpación de sus tierras; 2) en los años setenta surge desde un enfoque de mantenimiento de las lenguas indígenas y de sus hablantes -porque el aprendizaje de la lengua hegemónica (castellano, portugués, inglés y francés) no fue suficiente para superar procesos de exclusión, discriminación y racismo; y 3) a partir de los noventa, con las demandas sociales indígenas surge un lenguaje común en Latinoamérica y se habla de educación intercultural, que busca un lugar en la estructura del sistema educativo y una incorporación de saberes y conocimientos propios de las familias y comunidades al currículum escolar (Quintriqueo y Muñoz, 2015; Quintriqueo y Quilaqueo, 2019). En las tres etapas se observa que la Educación Intercultural Bilingüe (EIB) es demandada, planificada y desarrollada en función de los intereses de los estados, por lo que está dirigida sólo a estudiantes de ascendencia indígena o que están arraigados a familias y comunidades rurales.

En Chile, desde 1996 se implementa la EIB amparada en la Ley Indígena No. 19.253 (de 1993) como una forma de dar respuesta a las demandas indígenas, respecto a una educación contextualizada que considere la enseñanza de la lengua vernácula, los saberes y conocimientos educativos propios, para mejorar el éxito escolar y educativo de los estudiantes. El éxito escolar refiere a la aprobación de contenidos escolares que son certificados oficialmente por la escuela. Mientras que el éxito educativo, refiere al desarrollo integral del estudiante, para desenvolverse adecuadamente en el marco de la sociedad y la cultura actual (AriasOrtega, 2019). En este contexto, en Chile surgió entre 1996 y 2010 la figura del educador tradicional (considerado un sabio mapuche), que se incorpora a la educación escolar para enseñar la lengua y cultura propia, en colaboración con el profesor mentor. Su incorporación al aula se justifica debido al desconocimiento de los profesores de los saberes y conocimientos educativos mapuches, lo que limita la implementación de la ElB. Para ello, se conformó una nueva relación educativa, constituida por el profesor mentor y el educador tradicional, para la enseñanza escolarizada de la lengua y la cultura mapuche. 
El objetivo de este artículo es exponer los desafíos de la relación educativa en la incorporación de la episteme indígena en la EIB, como aportes epistémicos de métodos, saberes y conocimientos educativos mapuches posibles de incorporar en sus prácticas pedagógicas, desde un enfoque educativo intercultural.

\subsection{Racismo institucionalizado en la educación escolar}

El racismo institucionalizado en el sistema educativo escolar también subyace en la política de educación intercultural. Según Mampaey y Zanoni (2015), el racismo institucionalizado se relaciona con tres factores: 1) la práctica de educación monolingüe en contextos de colonización, donde la única lengua aceptada es la oficial; 2) la exclusión de las minorías religiosas en el medio escolar; y 3) el uso de dispositivos curriculares para transmitir la ideología nacionalista y el conocimiento eurocéntrico occidental. En el contexto latinoamericano es posible visualizar que en la institución escolar existe un racismo sistémico que se expresa en: 1) un currículum escolar de corte eurocéntrico occidental, que invisibiliza la episteme indígena en los contenidos escolares a enseñar (Quintriqueo y Quilaqueo, 2019); 2) la educación escolar se desarrolla desvinculada de los intereses de la familia y la comunidad indígena, la que no participa en la co-gestión de la enseñanza y el aprendizaje de sus hijos (Arias-Ortega, 2019); 3) los Programas Curriculares ofrecen una educación escolar homogeneizante y única para la formación de las nuevas generaciones, en la que se busca de manera consciente o inconsciente eliminar "el ser indígena" en los estudiantes (Luna y Contreras, 2019); y 4) los métodos educativos no consideran otras formas de enseñar y aprender, que están presentes en la pedagogía y educación indígena, los que son posibles de articular con el conocimiento y métodos educativos escolares, para pensar la educación escolar desde una perspectiva de interculturalidad crítica (Arias-Ortega, Quintriqueo y Valdebenito, 2018).

En ese contexto de exclusión y racismo en el marco de la educación escolar se produce una serie de procesos de minorización e invisibilización de la episteme indígena, demarcando así los tipos de relación educativa que establecería el indígena respecto de los no indígenas (Velasco, 2016). De este modo, la colonización del territorio latinoamericano ha traído consigo un proceso de violencia asociada a una colonialidad del saber, del ser y del poder hacia los pueblos indígenas, lo que se ha llevado a cabo a través del racismo biológico, epistémico e institucional.

El racismo biológico, entendido como la ideología de "división de razas", donde se les atribuye a los seres humanos un valor social de jerarquía o subalternidad, dependiendo de la "raza" de la que proviene el individuo, es el caso de los pueblos indígenas a quienes se les atribuye un déficit cognitivo y emocional (Paillalef, 2018). Es lo que ocurre en la relación educativa cuando profesores atribuyen menores expectativas de éxito escolar y educativo a los estudiantes indígenas, por considerar que carecen de capacidades cognitivas.

El racismo epistémico, entendido como la legitimización del conocimiento eurocéntrico occidental, como el único tipo de pensamiento capaz de producir conocimiento universal, verdadero y racional, para el desarrollo de la sociedad, el que es transmitido en la educación escolar (Mejía, 2015). Este racismo en la educación escolar se refleja en la instalación de un currículum que establece contenidos escolares como saber oficial, para la formación de las nuevas generaciones (Luna y Contreras, 2019; Velasco, 2016).

El racismo institucionalizado en el sistema educativo escolar chileno se ha mantenido arraigado a la estructura colonial de la escuela, mediante un currículum escolar monocultural eurocéntrico occidental y prácticas pedagógicas basadas en la hegemonía del conocimiento escolar (Arias-Ortega et al., 2018). Aquí, el racismo institucionalizado es entendido como las expresiones y prácticas racialmente diferenciadas al interior de la estructura de la organización escolar, las que inciden en la interacción comunicativa entre el profesor y los estudiantes (Castillo y Caicedo, 2010). De esta forma, en la educación escolar se rechaza el conocimiento indígena, negando su episteme propia, en las relaciones educativas entre profesores, estudiantes, miembros de la familia y comunidad en la implementación de la EIB.

En el marco de la EIB, la relación educativa entre el profesor y el educador tradicional se caracteriza por tensiones epistemológicas, producto de concebir la enseñanza de la lengua y la cultura desde dos epistemes distintas. Así, para el profesor mentor la enseñanza debe realizarse en la sala de clases, con base 
en los contenidos escolares propuesto en el marco del currículum escolar; en tanto que para el educador tradicional la enseñanza de la lengua y la cultura mapuche debe realizarse en el contexto de la vida cotidiana, en donde el estudiante se involucre en actividades concretas con la comunidad (Arias-Ortega, 2019). Dichas tensiones epistemológicas nos permiten sostener que ambas lógicas de pensamiento implican contrastes y desacuerdos en la forma de concebir la educación, los contenidos, finalidades y métodos educativos, desde la episteme indígena y escolar. Para superar estas tensiones en la implementación de la EIB es necesario avanzar en la deconstrucción de una relación educativa hegemónica de carácter monocultural, la que permita situarnos en una postura de apertura con el otro, para co-construir con los actores del medio educativo y social, desde una relación educativa intercultural.

\subsection{Relación educativa en perspectiva intercultural}

La relación educativa entre el profesor mentor y el educador tradicional constituye el encuentro entre un sujeto que enseña y otro que aprende en reciprocidad, donde el intercambio de roles, para enseñar la lengua y la cultura mapuche en el aula, debe ser sistemático (Arias-Ortega, 2019). En el contexto escolar la relación educativa refiere al encuentro intencional entre el profesor mentor, el educador tradicional y el estudiante, donde se producen experiencias orientadas a informar y formar sistemáticamente al otro en el ámbito objetivo y subjetivo del saber (Ouellet, 2015). La relación educativa entre profesores y estudiantes de ascendencia indígena, históricamente, se ha caracterizado por sustentarse en prejuicios, racismo y discriminación. Es lo que hemos constatado a nivel internacional, por ejemplo, en estudios realizados en Nueva Zelanda, Australia, México y Canadá, donde son los estudiantes indígenas quienes representan una menor escolarización y mayor deserción escolar (Bishop et al., 2012; Dietz, 2014).

En ese sentido, la relación educativa es un factor principal para revertir esta situación histórica de marginación del indígena en el contexto escolar. De esta manera, en la relación educativa el aprendizaje y la comunicación se constituyen en dos pilares esenciales para el aprendizaje, que promueve la implicación recíproca en el proceso de enseñanza-aprendizaje, y la relación educativa se constituye en un componente pedagógico esencial para la formación del estudiante que aprende, con base en distintas disciplinas y reglas básicas para descubrir su vida (Serina-Karsky, 2019). Así, la relación educativa implica que tanto el profesor mentor como el educador tradicional sean conscientes de la necesidad de apertura al otro para debatir $y$, en conjunto, construir un aprendizaje en común.

En la EIB la relación educativa en perspectiva intercultural demanda avanzar en un inter-aprendizaje entre el conocimiento indígena y escolar para la enseñanza de la lengua y la cultura mapuche en la escuela. Coincidimos con Ouellet (2015) y Serina-Karsky (2019) en que una relación educativa en perspectiva intercultural debiese reconocer los saberes mapuches, para reafirmar el diálogo intraétnico e interétnico en la educación escolar. Esto permitiría contribuir con propuestas de contenidos educativos para resolver problemas locales y globales como el racismo, prejuicio y minorización, desde epistemologías propias en el marco de la EIB (Dietz y Mateos, 2011; Valladares y Olivé, 2015). Esta relación educativa intercultural basada en el inter-aprendizaje para reflexionar sobre los conocimientos educativos mapuche y el conocimiento escolar permitiría fortalecer el pensamiento y la práctica educativa, para construir procesos de enseñanzaaprendizaje contextualizados a la realidad social y cultural de los estudiantes. Lo anterior es posible mediante la implicación de los estudiantes, el profesor, la escuela, la familia y la comunidad en la educación escolar. Esta implicación considera la intervención y desarrollo de proyectos educativos institucionales, desde un enfoque co-constructivo, que permita indagar y sistematizar saberes y conocimientos educativos mapuches y escolares para ser incorporados a los procesos de enseñanza-aprendizaje desde un enfoque educativo intercultural.

\section{Método}

La metodología empleada es la investigación educativa, que busca comprender los desafíos de la relación educativa en la implementación de la EIB (Gauthier y Bourgeois, 2016). La técnica empleada para la selección de los participantes es intencional no probabilística (Gauthier y Bourgeois, 2016). El estudio consideró cuatro escuelas municipales de Educación Básica en el ámbito rural, situadas en comunidades mapuches de la comuna de Padre Las Casas, en La Araucanía. Los criterios de inclusión fueron: 1) escuelas 
donde se implementa la EIB con la figura de un profesor mentor y un educador tradicional; y 2) escuelas situadas en Padre Las Casas. Por lo tanto, se contó con la participación de 8 sujetos, 4 profesores mentores y 4 educadores tradicionales. Los profesores mentores que participaron en el estudio han trabajado durante 8 años en el establecimiento en la implementación de la EIB, no son de ascendencia indígena y su edad fluctúa entre los 35 a 50 años. Los educadores tradicionales han trabajado 5 años en el establecimiento enseñando la lengua y la cultura mapuche; son de ascendencia mapuche y su edad fluctúa entre 45 a 60 años. La técnica de recolección de información fue la entrevista semi-dirigida que indaga sobre: 1) la relación educativa entre el profesor mentor y educador tradicional; 2) formas en que se incorporan saberes y conocimientos educativos mapuches a la educación escolar; y 3) racionalidad del conocimiento escolar y del conocimiento educativo mapuche que se incorporan a las prácticas pedagógicas.

Para el análisis se empleó la teoría fundamentada, que busca generar categorías centrales a partir de los datos obtenidos en la codificación abierta y axial (Gauthier y Bourgeois, 2016). Para el análisis de la información se empleó el análisis de contenido que busca develar núcleos de temas de orden abstracto, dotados de sentido, desde la perspectiva de los productores del discurso, a partir de contenidos concretos expresados de forma explícita y latente en el texto (Gauthier y Bourgeois, 2016). Esto, en complementariedad con la teoría fundamentada, que busca generar categorías centrales a partir de los datos obtenidos en la codificación abierta y axial (Gauthier y Bourgeois, 2016). En la codificación se utilizó la siguiente nomenclatura: 1) profesor mentor (PM); y 2) educador tradicional (ET) y se incorpora entre paréntesis cuadrado el número en el cual se encuentra la expresión del profesor mentor en la unidad hermenéutica de Atlas ti 5.0, por ejemplo: (PM6 [233:233]). Esta lógica de codificación se aplica también para las entrevistas realizadas a los educadores tradicionales.

\section{Resultados}

El análisis de las entrevistas aplicadas tanto a los profesores mentores como a los educadores tradicionales dio como resultado un total de 330 recurrencias. Las categorías de contenidos centrales que emergen con mayor recurrencia en el testimonio de los participantes son: 1) Métodos educativos mapuches asociados a un $57.2 \%$ de las recurrencias (189) y son definidos como herramientas culturales que orientan la formación de personas desde el marco social y cultural propio, para moverse tanto en la esfera social occidental como indígena (Quintriqueo y Quilaqueo 2019); y 2) Saberes y conocimientos educativos mapuches asociados a un $42.8 \%$ de las recurrencias (141) y está referido a contenidos educativos que han sido transmitidos de generación en generación, por los sabios de las familias, desde la memoria social. Precisamos que los sabios mapuches no necesariamente tienen un rol sistemático en la estructura de la familia y comunidad, sino que se recurre a ellos para solicitar apoyo en situaciones puntuales. Por ejemplo, para enseñar saberes y conocimientos sobre prácticas socio-religiosas en la familia y la comunidad.

\subsection{Métodos educativos mapuches}

En la Tabla 1 se presenta la categoría Métodos educativos mapuches con sus respectivos códigos, recurrencias y porcentajes. 
Tabla 1. Métodos educativos mapuches

\begin{tabular}{lcc}
\hline Códigos & Recurrencia & Porcentaje \\
\hline Aprender escuchando & 46 & 24.3 \\
Aprender observando & 29 & 15.3 \\
Aprender haciendo & 28 & 14.8 \\
Mapunzugun & 23 & 12.1 \\
Inatuzugu (indagación) & 20 & 10.5 \\
Güxam (conversación) & 15 & 7.9 \\
Epew (relato fundacional) & 15 & 7.9 \\
Yamuwün (respeto a las personas) & 13 & 6.8 \\
\hline Total & 189 & 100 \\
\hline
\end{tabular}

Figura 1. Métodos educativos mapuches

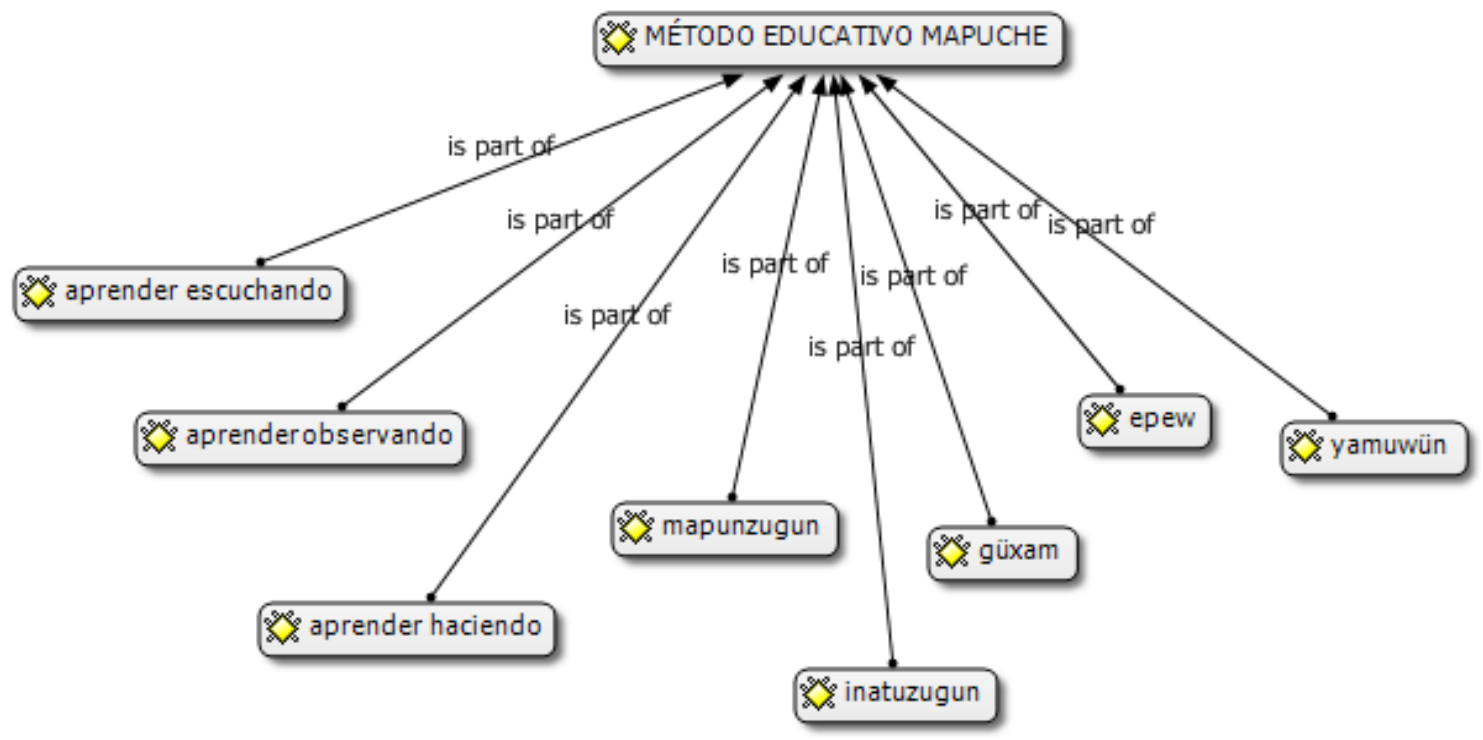

En la red conceptual No. 1 se presenta la categoría asociada a métodos educativos mapuches que se compone de 8 códigos, los que se presentan de forma sistemática, de mayor a menor recurrencia, según el discurso de los participantes. Cada código se complementa en el análisis con la incorporación de testimonios más representativos.

La Figura 1 ilustra los códigos que se configuran en las formas de educar a los niños y jóvenes mapuches en relación con prácticas epistémicas propias. Las prácticas epistémicas son acciones que se sustentan en una estructura propia del conocimiento, lo que permite dar sentido y significado a la realidad natural, social, cultural y espiritual. Así, el aprender escuchando obtiene una frecuencia de 24.3\% de un total de 189 recurrencias, constituyéndose en un método educativo reconocido en el testimonio de los educadores tradicionales, para formar a los niños en los conocimientos sociales, naturales, culturales y espirituales, desde la educación familiar. En este método educativo los sabios son los agentes reconocidos por la comunidad, como sujetos que transmiten la memoria social mapuche, debido a sus experiencias de vida en relación con los saberes y conocimientos educativos propios. Un educador tradicional: 
[señala la mamá] nos conversaba (...), nos explicaba qué teníamos que hacer, nos decía qué había que consumir: poñü (papas), kachija (trigo) [recolectar] changle (hongo comestible) y napor (vegetal comestible) ... (ET6 [233:233]).

El relato del educador tradicional da cuenta de la importancia de desarrollar la habilidad de escuchar, ya que mediante esta práctica epistémica se adquieren los conocimientos educativos mapuches de la tradición oral.

El Aprender observando tiene una frecuencia de $15.3 \%$ en el discurso de los educadores tradicionales, que permite a los niños y jóvenes mapuches aprender y comprender los conocimientos educativos propios, mediante su participación en prácticas socioculturales y en la relación con la naturaleza. Al respecto, un educador tradicional señala:

Esos conocimientos los fui viviendo, nos criamos así, íbamos a los geykurewen que es una ceremonia para el cambio de rewe (altar de la machi). Ahí vivíamos y experimentábamos nuestros conocimientos. Nadie nos enseñaba, mirábamos y los íbamos aprendiendo... (ET6 [62:62]).

El testimonio nos plantea la necesidad de que los estudiantes ejerciten la observación detallada de las actividades propias de su medio social, natural, cultural y espiritual.

El Aprender haciendo tiene una frecuencia de $14.8 \%$ y refiere a la participación directa de los niños en el quehacer cotidiano de la familia. Mediante este método se busca que los niños y jóvenes mapuches se impliquen en prácticas socioculturales y en actividades de la vida cotidiana de forma autónoma. Por ejemplo, cuidar los animales y el hilado de lana, para que los niños y jóvenes logren comprender los conocimientos y contenidos educativos que le subyacen. Al respecto, un educador tradicional menciona:

(...) [A los estudiantes se les podría dar] la oportunidad de ir a los gijatunes (rogativa), [para] practicar los conocimientos que allí aparecen. Allí, ellos pueden conocer por qué se hace el gijatun, cuál es el sentido, el por qué bailar con unas ramas. Los niños [tendrían] la oportunidad de observar y experimentar todos esos conocimientos, en la medida que participen en las ceremonias de la comunidad... (ET6 [249:249]).

De acuerdo al testimonio, el método Aprender haciendo facilita a los niños y jóvenes la experimentación para el aprendizaje, de una manera más dinámica y acorde al marco de referencia propio de los estudiantes.

El código mapunzugun obtiene una frecuencia de 12.1\%, asociado a la oralidad para la transmisión de los saberes y conocimientos educativos propios. Al respecto se señala: "(...) Yo creo que la enseñanza de los conocimientos mapuches debería ser en mapunzugun, eso es importantísimo..." (ET6 [294:294]). En la escuela a los niños se les debe hablar en mapunzugun para revertir la situación de abandono y desconocimiento de la lengua vernácula. Tal como se observa en el siguiente discurso:

Chumkaw no rume kimlayay mapunzugun pu püchike che, ruka mew enseñanza elugepe (los niños nunca van a aprender bien el mapunzugun si los papás no le enseñan en la casa), ruka mew azümelgele pu püchike che (los niños desde la casa deberían venir ya con ese conocimiento), [pero eso no ocurre], los padres jóvenes (...) no saben hablar (kimlay mapunzugun egün) ... (ET1 [299:299+]).

De acuerdo al testimonio, en la actualidad los padres no enseñan el mapunzugun a los niños en la familia, debido a su desconocimiento de la lengua. En el discurso del educador tradicional es primordial que los niños aprendan el mapunzugun en el contexto familiar, para reforzar su enseñanza-aprendizaje en la educación escolar.

El inatuzugu obtiene una frecuencia de $10.5 \%$, y corresponde a una forma de aprender indagando en el contexto social, natural, cultural y espiritual. Al respecto, un educador tradicional señala: 
[Antes] se aprendía mediante el inatuzugu, el niño acompañaba a su familia (...) en distintas actividades, puede ser en un xawün (reunión), en el trabajo, esa era la forma de adquirir el tuwün (conocimiento sobre la ascendencia parental paterna). (PM8 [73:73]).

Así, mediante la indagación se adquieren los conocimientos propios.

El güxam obtiene una frecuencia de 7.9\% y corresponde a la práctica de la conversación entre un adulto, los niños y jóvenes en el contexto familiar y comunitario. Con relación al güxam, un educador tradicional señala: "Le enseñamos a los niños, lo que a mí me enseñaron mis padres, que no deben ir al mediodía al río, que no deben andar jugando afuera (...), porque en ese horario anda el weza kürüf (fuerza negativa) (...). (ET1 [41:41]). ${ }^{2}$

Según el testimonio, a través de la conversación los niños aprenden a mantener una relación de equilibrio con el medio ambiente.

El epew obtiene una frecuencia de $7.9 \%$ y corresponde a un relato fundacional construido por los mapuches para transmitir contenidos educativos a los niños y jóvenes de forma lúdica, para el aprendizaje procedimental y actitudinal. Los epew generalmente representan situaciones en relación con animales, en donde la historia y sus comportamientos están asociados a características que pueden adquirir los seres humanos, mediante el aprendizaje central que se busca construir en los niños y jóvenes. Al respecto, un profesor mentor señala: "Los epew, son relatos que dejan una enseñanza (...). (PM2 [37:37])", y agrega: "El epew es un relato que yo lo invento o lo trato de hacer (...). (ET4 [191:191]).

De acuerdo a los testimonios, mediante los epew se logra transmitir valores que promueven el buen comportamiento, para ser buenas personas.

El yamuwün obtiene una frecuencia de $6.8 \%$ y refiere al respeto como valor educativo necesario de construir y practicar en la interacción social de niños y jóvenes, así como sobre la relación que se establece con el medio natural y espiritual. También permite transmitir el respeto a los parientes cercanos y a las personas de la comunidad. Al respecto, un educador tradicional comenta: "A los niños mapuches lo primero que se les enseña es el respeto, el respeto a los mayores, el respeto a una dama, el respeto a todas las personas (ET7 [165:165])".

En relación con los testimonios, mediante el yamuwün se enfatiza en la necesidad de formar la identidad de los niños y una forma de actuar en el medio social y cultural.

En síntesis, los métodos educativos mapuches constituyen prácticas epistémicas que se activan mediante la implicación de las personas en la relación con el medio natural, social cultural y espiritual.

\subsection{Saberes y conocimientos educativos mapuches}

La Tabla 2 presenta los códigos, recurrencias y porcentajes asociados a la categoría Saberes y conocimientos educativos mapuches.

Tabla 2. Saberes y conocimientos educativos mapuches

\begin{tabular}{lcc}
\hline Códigos & Recurrencia & Porcentaje \\
\hline Relación hombre-naturaleza & 88 & 62.4 \\
Conocimiento territorial & 29 & 20.1 \\
Ubicación espacio-temporal & 24 & 17.2 \\
\hline Total & 141 & 100 \\
\hline
\end{tabular}

${ }^{2}$ Cuando sólo se utilizan testimonios de educadores tradicionales es porque los profesores mentores no expresaron conocimientos sobre el respectivo código. 
Figura 2. Saberes y conocimientos educativos mapuches ${ }^{3}$

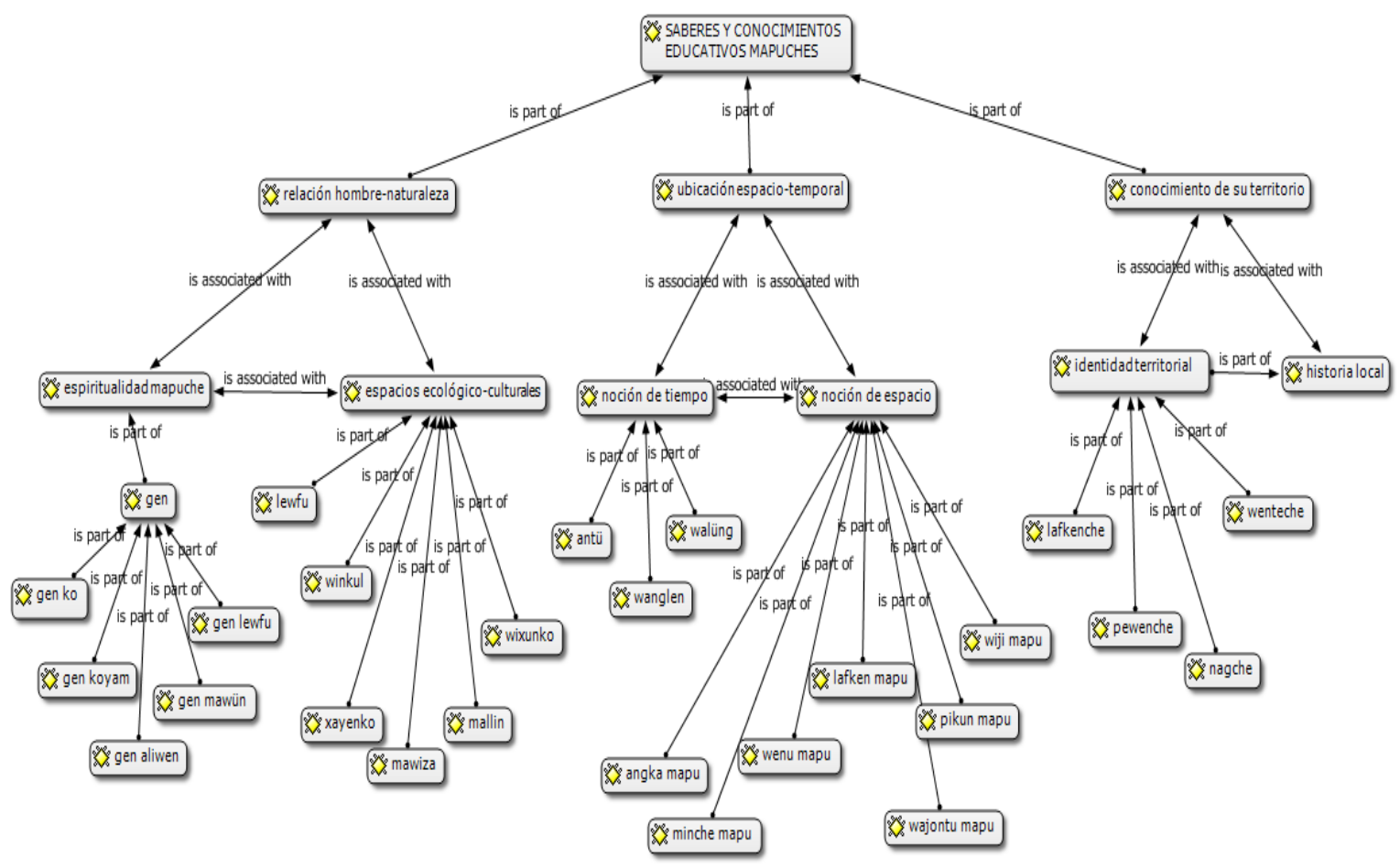

La figura 2 se compone del código relación hombre-naturaleza, que obtiene una frecuencia de $62.4 \%$ de un total de 141 recurrencias. Dicho código está asociado a la importancia de mantener una relación de respeto y equilibrio con las fuerzas espirituales que habitan y cuidan los espacios ecológicos culturales. Al respecto se señala:

Esos gen son los espíritus de la naturaleza. Nosotros como mapuche tenemos otra visión, creemos que la tierra nunca está sola, eso es parte de lo que nos regaló la naturaleza, nuestro Günechen (Ser Superior). (...) Es por eso que [cuando ingresamos a un monte] debemos pedir permiso, para poder entrar y caminar tranquilo [en los distintos lugares]... (ET7 [171:171]).

Lo anterior es reforzado por un testimonio que plantea: “... nosotros le llamamos gen mapu, dueño de la tierra; gen koyam, dueño del roble; gen ko, dueño del agua; gen mawün, dueño de la lluvia, gen lewfü, dueño de los ríos (...)" (ET4 [277:277]). De acuerdo con el testimonio podemos inferir que los gen refieren a fuerzas espirituales que resguardan una relación de equilibrio del hombre con su entorno natural.

En relación con el código Conocimiento territorial obtiene una frecuencia de $20.1 \%$ y refiere a los conocimientos educativos sobre el territorio, que han sido construidos mediante la relación directa del hombre con su medio social, natural, cultural y espiritual. Según los testimonios, los conocimientos territoriales varían dependiendo de la identidad territorial a la cual se pertenezca:

En este territorio [wenteche] (gente del valle central), no se hacen esos bailes, aquí no se baila, porque el lafkenche (gente del borde costero) tiene como escudo el avestruz y en relación a ella hacen el baile. En cambio, aquí hacemos el purrün (baile) alrededor del rewe y los caballos danzan

\footnotetext{
${ }^{3}$ Esta categoría emerge sólo desde el testimonio de los educadores tradicionales, puesto que son ellos quienes poseen el dominio de saberes y conocimientos educativos mapuches, producto de su educación familiar.
} 
alrededor del rewe, en círculos, y vienen terminando el día, van dándole gracias al sol... (ET1 [155:155]).

El código Ubicación espacio-temporal obtiene una frecuencia de $17.2 \%$ y refiere a las nociones de espacio vertical y horizontal que determina la relación del hombre con el mundo. Al respecto un educador tradicional comenta:

Para nosotros los mapuches debajo de la tierra hay vida, a lo mejor para los wigkas debajo de la tierra no hay vida, [puede ser] algo sin sentido, quizás algo muerto. Por ejemplo, en el angka mapu (espacio vertical intermedio inferior) también hay vida, o lo que significa el wajontu mapu (mundo) que para nosotros los mapuches es vida, o sea todo tiene vida y todo tiene su espacio. [Con respecto al] cielo, tenemos el [wenu mapu], angka mapu. También tenemos münche mapu (espacio vertical inferior), waj mapu (macro-territorialidad mapuche) que es el espacio donde estamos nosotros (ET6 [132:132]).

Es así como las nociones de tiempo-espacio se ha construido desde el conocimiento mapuche para organizar la vida social, cultural, económica y espiritual. Al respecto, el testimonio de un educador tradicional menciona: “...Tampoco deben salir en la noche a jugar al patio. Yo [a mis hijos] no los dejaba salir, porque en esos horarios se tenía el conocimiento de que andaba el anchümalen (fuerzas espirituales) (...)" (ET1 [41:41]). Estas nociones de tiempo-espacio también están asociadas a actividades que se realizan en la comunidad.

En general, los resultados muestran que los profesores mentores desconocen las prácticas epistémicas mapuches, no poseen un conocimiento ni oral ni escrito sobre el mapunzugun, lo que limita el desarrollo de la EIB. Esto se refleja en la baja recurrencia de contenidos educativos mapuches que emergen del análisis en las entrevistas de los profesores mentores. En cambio, en el análisis de las entrevistas de educadores tradicionales se constata un dominio sobre el mapunzugun asociado a métodos y saberes educativos mapuches, que se constituyen en aportes epistémicos para la implementación de la EIB. Sin embargo, la brecha en el dominio de los saberes y conocimientos educativos mapuches entre los profesores mentores y educadores tradicionales limita la interacción y colaboración entre ambos agentes educativos, afectando de forma negativa la relación educativa intercultural.

\section{Discusión y conclusiones}

Los principales puntos de discusión que emergen son: 1) métodos educativos; 2) saberes y conocimientos educativos; 3) desvalorización de los conocimientos educativos mapuches en la educación escolar; y 4) aportes epistémicos de métodos y conocimientos educativos mapuches susceptibles de incorporar al currículum escolar, desde un enfoque educativo intercultural.

Respecto de los métodos educativos mapuches, se constata el reconocimiento de los sabios como agentes educativos que poseen conocimientos en la memoria social, producto de su experiencia de educación familiar. Esto es consistente con la literatura científica, que sostiene que en los pueblos indígenas los sabios son los depositarios de los métodos educativos para formar a las nuevas generaciones (Cajete, 1994; Quintriqueo y Quilaqueo, 2019). En esta perspectiva, la implementación de la EIB no sólo requiere de la intervención del profesor mentor y del educador tradicional, sino también de la colaboración activa de los sabios mapuches que permitan profundizar la formación de persona, recurriendo a la episteme propia que sustenta los métodos educativos.

Los métodos educativos mapuches permiten desarrollar habilidades (aprender escuchando, observando, haciendo y el inatuzugu) para reforzar y enriquecer el dominio disciplinario en la educación escolar. Por ejemplo, en actividades de experimentación, comprensión y explicación de hechos, fenómenos y procesos en asignaturas como Ciencias Naturales, Historia, Tecnología y Artes. Estudios empíricos remarcan la importancia de considerar dichos métodos de experimentación para contextualizar la educación en contexto indígena e intercultural (Quintriqueo y Quilaqueo, 2019). Asimismo, se constata que existen métodos educativos para apoyar el desarrollo de la inteligencia emocional desde la cosmovisión mapuche 
(la oralidad-mapunzugun, güxam, epew, yamuwün). En las Américas existen evidencias empíricas sobre la relevancia de incorporar dichos métodos a la educación escolar, lo que potenciaría la formación integral de estudiantes indígenas y no indígenas, asegurando el arraigo social, cultural y territorial (Cajete, 1994; Somerville, 2011). Esto implica que en la relación educativa entre profesores mentores y educadores tradicionales se consideren métodos educativos mapuches para la implementación de la ElB. Es decir, favorecer la experimentación de los estudiantes en el medioambiente, como condición primordial para poner en práctica métodos educativos mapuches en la educación intercultural. En este sentido, para enseñar la lengua indígena en la escuela, es esencial recurrir a prácticas epistémicas que permitan vincular a los sujetos con la memoria social y el medio ambiente.

En esa perspectiva, constatamos que en la relación educativa intercultural se requiere de un método de coconstrucción como condición para la enseñanza-aprendizaje, desde un enfoque educativo intercultural. Esto releva la importancia de la educación familiar para apoyar la relación educativa entre el profesor mentor, el educador tradicional y los estudiantes, lo que permita formar a los estudiantes con base en métodos educativos propios, desde una relación de equilibrio entre las personas y su medio ambiente en las respectivas territorialidades. Esto debido a que los métodos educativos escolares, en general, no responden a las prácticas epistémicas mapuches (Valdebenito, 2017). En efecto, es urgente implicar a la familia y la comunidad mapuche en la concepción, implementación y evaluación de proyectos educativos en perspectiva intercultural, lo que permita mejorar de manera sustantiva la implementación de la EIB.

Con relación a la categoría Saberes y conocimientos educativos mapuches, constatamos que la oralidad es un mecanismo de construcción y transmisión de conocimientos, en el marco de la educación familiar, en cuyo proceso los sabios cumplen un rol de vital importancia. En este sentido, el rol atribuido al educador tradicional en la escuela es reemplazar la función de los padres y sabios mapuches. Sin embargo, los resultados de investigaciones empíricas muestran que los educadores tradicionales, en general, carecen del dominio de los métodos, saberes y conocimientos educativos mapuches (Quintriqueo y Quilaqueo, 2019). Una posible solución a este problema es la desescolarización de las prácticas de enseñanzaaprendizaje de los profesores mentores y educadores tradicionales en la implementación de la ElB. En efecto, se requiere que el educador tradicional recurra sistemáticamente a quienes tienen un mayor dominio del conocimiento que se enseña en la educación escolar, sea este un sabio o padre de familia que domina un oficio, para apoyar los procesos educativos desde un enfoque de co-construcción. Esto implica situar la implementación de la EIB en la lógica de experimentación y el desarrollo de la inteligencia emocional indígena para asegurar el éxito escolar y educativo de todos los estudiantes.

En la investigación se constató que existen saberes y conocimientos educativos mapuches susceptibles de ser incorporados a la implementación de la EIB. En este sentido, existen evidencias empíricas sobre la existencia de dichos conocimientos indígenas y su relevancia de ser incorporado en el currículum escolar desde un enfoque educativo intercultural (Sartorello, 2014). Por ejemplo, constatamos que en el contexto de las escuelas de La Araucanía existen espacios ecológicos culturales que se constituyen en un laboratorio natural para experimentar el conocimiento educativo. Desde la lógica del conocimiento educativo indígena, dichos espacios de aprendizaje son pertinentes para el aprendizaje de los aspectos espirituales, para construir el pensamiento de reciprocidad del hombre con la naturaleza.

Sostenemos, que en la educación escolar es posible revertir la desvalorización que existe hacia los saberes y conocimientos educativos mapuches. Esto, mediante el empoderamiento del educador tradicional quien debiera ser capaz de implicar al profesor mentor en la incorporación de los saberes y conocimientos educativos mapuches en sus prácticas pedagógicas. Lo anterior, permitiría a la vez, establecer una verdadera relación educativa intercultural, sustentada en un enfoque de co-construcción de la enseñanza y el aprendizaje en escuelas situadas en contextos indígenas.

Sin embargo, este proceso de incorporación de conocimientos indígenas a la educación escolar no es suficiente porque en contexto de colonización implica superar la colonialidad del saber y el orden epistémico del conocimiento occidental en la escuela (Pérez y Argueta, 2011). En contexto mapuche el desafío de la relación educativa entre el profesor mentor y el educador tradicional es enseñar la lengua y cultura mapuche desde su propia estructura epistémica. Esto implica que el profesor mentor (sea mapuche 
o no) valore y valide el conocimiento indígena en la implementación de la EIB.

Los resultados de la investigación dan cuenta de los aportes epistémicos que los métodos, saberes y conocimientos educativos mapuches, pudiesen aportar en la educación escolar. A la vez, nos plantea el desafío de concretizar su incorporación al currículum escolar, desde un enfoque educativo intercultural, para pensar la formación de las nuevas generaciones desde una mirada de descolonización del saber.

Una posible solución implicaría avanzar en procesos de co-construcción de un currículum intercultural. Según Sartorello (2014), esto implica construir un currículum escolar que considera necesaria la vinculación con los miembros de la comunidad en la que se encuentra inserta la escuela para que, en conjunto, indaguen, sistematicen y co-construyan actividades de enseñanza-aprendizaje que fortalezcan, por una parte, el desarrollo de la identidad sociocultural y territorial de los estudiantes, y por otra, la articulación de conocimientos indígenas y escolares para proponer acciones educativas contextualizadas a la territorialidad de los estudiantes.

Llegamos a la conclusión de que la implementación de la EIB requiere de una sensibilización y formación continua tanto de los profesores mentores como de los educadores tradicionales para aceptar y validar otras epistemes, diferente a la occidental, para sustentar sus prácticas educativas. Dicha sensibilización y formación continua plantea el desafío de incorporar los aportes epistémicos de métodos, saberes y conocimientos educativos mapuches a la educación escolar, desde un enfoque educativo intercultural. Por ejemplo, mediante la incorporación del inatuzugu como práctica epistémica que favorezca la implementación de la EIB, a través de procesos de indagación desde el conocimiento propio y el conocimiento escolar, para la enseñanza de las ciencias naturales y sociales.

\section{Referencias}

Arias-Ortega, K. E. (2019). Relación educativa entre el profesor mentor y el educador tradicional en la educación intercultural (Tesis Doctoral). Universidad Católica de Temuco, Chile.

Arias-Ortega, K. E., Quintriqueo, S. E. y Valdebenito, V. H. (2018). Monoculturalidad en las prácticas pedagógicas en la formación inicial docente en La Araucanía, Chile. Educação e Pesquisa, 44 (e164545). https://doi.org/10.1590/s1678-4634201711164545

Bishop, P. R., Berryman, M. A. Wearmouth, J. B., Peter, M. y Clapham, S. (2012). Professional development, changes in teacher practice and improvements in Indigenous students' educational performance: a case study from New Zealand. Teaching and Teacher Education, 28(5), 694-705.

https://doi.org/10.1016/j.tate.2012.02.002

Caiceo, J. (2011). Desarrollo de la educación parvularia en Chile. Revista Historia da Educação, 15(34), 22-44. https://dialnet.unirioja.es/servlet/articulo?codigo=4891629

Cajete, G. (1994). Look to the mountain: an ecology of Indigenous education. Kivaki Press.

Castillo, E. G. y Caicedo, J. A. (2010). Las luchas por otras educaciones en el bicentenario: de la iglesiadocente a las educaciones étnicas. Revista Nómadas, (33),109-12.

http://www.scielo.org.co/scielo.php?script=sciarttext\&pid=S0121-75502010000200009\&lng=en\&tlng=es

Dietz, G. (2014). Educación intercultural en México. CPU-e, Revista de Investigación Educativa, (18), 162-171. https://doi.org/10.25009/cpue.v0i18.761

Dietz, G. y Mateos, L. S. (2011). Interculturalidad y educación intercultural en México: un análisis de los discursos nacionales e internacionales en su impacto en los modelos educativos mexicanos. Secretaría de Educación Pública-Coordinación General de Educación Intercultural y Bilingüe. 
Fajardo, D. M. (2011). Educación intercultural bilingüe en Latinoamérica: un breve estado de la cuestión. LiminaR. Estudios Sociales y Humanisticos, 9(2), 15-29. https://doi.ora/10.29043/liminar.v9i2.45

Gaete, M. y Morales, R. (2011). Articulación del sistema de educación superior en Chile: posibilidades, tensiones y desafíos. Revista Calidad en la Educación, (35), 1-89. https://doi.org/10.31619/caledu.n35.96

Gasché, J. (2013). Éxitos y fracasos de una propuesta educativa basada sobre el método inductivo intercultural e implementada en el Perú, México y el Brasil. ISEES: Inclusión Social y Equidad en la Educación Superior, (13), 17-34. https://www.fundacionequitas.cl/publicaciones/isees/13/4421671.pdf

Gauthier, B. y Bourgeois, I. (2016). Recherche sociale de la problématique à la collecte des données. [Investigación social del problema a la recopilación de datos]. Presses de l'Université du Québec.

López, L. y Küper, W. (1999). La educación intercultural bilingüe en América Latina: balance y perspectivas. Revista Iberoamericana de Educación, (20), 17-85.

Luna, L. y Contreras, R. (2019). Educación mapuche y descolonización de la escuela: el análisis de un caso etnográfico de una escuela lafkenche de La Araucanía. En S. Quintriqueo y D. Quilaqueo (Eds.), Educación e interculturalidad: aproximación crítica y decolonial en contexto indígena (pp. 108-124). Universidad Católica de Temuco.

Mampaey, J. y Zanoni, P. (2015). Reproducing monocultural education: ethnic majority staff's discursive constructions of monocultural school practices. British Journal of Sociology of Education, 37(7), 928-946. https://doi.org/10.1080/01425692.2014.1001059

Mejía, J. (2015). Modernidad y conocimiento social: la emergencia de un discurso epistémico en América Latina. Cinta de Moebio, (54), 290-301. https://doi.org/10.4067/S0717-554X2015000300006

Ministerio de Educación de Chile (Mineduc). (2016). Estadísticas de la Educación 2016. Ministerio de Educación de Chile, Programa de Educación Intercultural Bilingüe.

Ouellet, S. (2015). Relations éducatives et apprentissage : regards diversifiés de professionnels en éducation et futurs chercheurs [Relaciones educativas y aprendizaje: diversas perspectivas de los profesionales de la educación y los futuros investigadores]. Presses de l’Université du Québec.

Paillalef, J. (2018). Los mapuches y el proceso que los convirtió en indios. Psicología de la discriminación. Catalonia.

Pérez, M. L. y Argueta, A. (2011). Saberes indígenas y diálogo intercultural. Cultura científica y saberes locales, 5(10), 31-56. http://www.culturayrs.unam.mx/index.php/CRS/article/viewFile/447/447

Quintriqueo, S. y Muñoz, G. (2015). Demandes educatives éducationelles sociohistoriques et éducation interculturelle en contexte mapuche. En R. Salas y F. Le Bonniec (Coords), Les mapuche á la mode. Modes d'existence et de résistance au Chili, en Argentine et au- delá [Los Mapuche a la moda. Modos de existencia y resistencia en Chile, Argentina y más allá] (pp. 95-122). L'Harmattan.

Quintriqueo, S. y Quilaqueo, D. (2019). Educación e interculturalidad: aproximación crítica y decolonial en contexto indígena. Universidad Católica de Temuco.

Quintriqueo, S., Morales, S., Quilaqueo, D. y Arias-Ortega, K. (2016). Interculturalidad en la formación inicial docente: invitación a construir un dialogo intercultural. Universidad Católica de Temuco.

Sartorello, S. (2014). La co-teorización intercultural de un modelo curricular en Chiapas, México. Revista Mexicana de Investigación Educativa, 19(60), 73-101. 
Serina-Karsky, F. (2019). Bien-être scolaire: des clés pour demain [Bienestar escolar: claves para el mañana]. L’Harmattan.

Somerville, M. (2011). Art, community and knowledge flows. En T. Fenwick y L. Farrell (Eds.), Knowledge mobilization and educational research: politics, languages, responsibilities (pp. 86-99). Routledge.

Valdebenito, X. (2017). Hacia una comprensión del vínculo entre las prácticas de enseñanza de educadores y educadoras tradicionales indígenas y el espacio escolar (Documento de trabajo No. 10). Centro de Estudios del Mineduc.

Valladares, L. y Olivé, L. (2015). ¿Qué son los conocimientos tradicionales? Apuntes epistemológicos para la interculturalidad. Cultura y Representaciones Sociales, 10(19), 61-101.

http://www.culturayrs.unam.mx/index.php/CRS/article/view/391/391

Velasco, S. (2016). Racismo y educación en México. Revista Mexicana de Ciencias Políticas y Sociales, 61(226), 379-408. http://www.revistas.unam.mx/index.php/rmcpys/article/view/53667 